\title{
Noise Robust Exemplar Matching with Alpha-Beta Divergence
}

\author{
Emre Yilmaz ${ }^{\mathrm{a}, *}$, Jort F. Gemmeke ${ }^{\mathrm{b}}$, Hugo Van hamme ${ }^{\mathrm{a}}$ \\ ${ }^{a}$ Dept. ESAT-PSI, KU Leuven, Kasteelpark Arenberg 10, B-3001, Heverlee, Belgium \\ ${ }^{b}$ Audience Inc., 331 Fairchild Drive, 94043, Mountain View, CA, USA
}

\begin{abstract}
The noise robust exemplar matching (N-REM) framework performs automatic speech recognition using exemplars, which are the labeled spectrographic representations of speech segments extracted from training data. By incorporating a sparse representations formulation, this technique remedies the inherent noise modeling problem of conventional exemplar matching-based automatic speech recognition systems. In this framework, noisy speech segments are approximated as a sparse linear combination of the exemplars of multiple lengths, each associated with a single speech unit such as words, half-words or phones. On account of the reconstruction error-based back end, the recognition accuracy highly depends on the congruence of the speech features and the divergence metric used to compare the speech segments with exemplars. In this work, we replace the conventional KullbackLeibler divergence (KLD) with a generalized divergence family called the Alpha-Beta divergence with two parameters, $\alpha$ and $\beta$, in conjunction with mel-scaled magnitude spectral features. The proposed recognizer traverses the $(\alpha, \beta)$ plane depending on the amount of contamination to provide better separation of speech and noise sources. Moreover, we apply our recently proposed active noise exemplar selection (ANES) technique in a more realistic scenario where the target utterances are degraded by genuine room noise. Recognition experiments on the small vocabulary track of the $2^{\text {nd }}$ CHiME Challenge and the AURORA-2 database have shown that the novel recognizer with the AB divergence and ANES outperforms the baseline system using the generalized KLD with tuned sparsity, especially at lower SNR levels.
\end{abstract}

Keywords: automatic speech recognition, noise robustness, exemplar matching, alpha-beta divergence, reconstruction error

\section{Introduction}

Data-driven automatic speech recognition (ASR) techniques (De Wachter et al., 2003; Aradilla et al., 2005; Deselaers et al., 2007; Sundaram and Bellegarda, 2012; Sainath et al., 2012; Heigold et al., 2012; Sun et al., 2014) became popular in the last decade as a viable alternative after the long dominance of statistical acoustic modeling in the form of the Gaussian mixture models (GMM) in hidden Markov models (HMM) (Bourlard et al., 1996). Templates or exemplars are labeled speech segments of multiple lengths extracted from training data, each associated with a certain class, i.e. a speech unit such as phones, syllables or words. As they preserve the complete duration and trajectory information, exemplars are more immune to the inherent spectrotemporal variation of speech and its deteriorating effect on the ASR (Benzeghiba et al., 2007) compared to the conventional GMM-HMM- or deep neural networks (DNN)-based recognition systems. Moreover, it has been shown that using reasonably large exemplar sets overcomes the well-known generalization problem of the previous exemplarbased approaches (Seppi and Van Compernolle, 2010; Sun and Zhao, 2011; Yilmaz et al., 2013a).

\footnotetext{
${ }^{*}$ Corresponding author, tel. $+32-16-321828$

Email addresses: emre.yilmaz@esat.kuleuven. be (Emre Y1lmaz), jgemmeke@amadana.nl (Jort F. Gemmeke), hugo.vanhamme@esat.kuleuven. be (Hugo Van hamme)
}

Exemplar matching-based recognition can be performed by evaluating the similarity of the exemplars with the segments from the input speech with respect to a distance/divergence metric by applying dynamic time warping (Sakoe and Chiba, 1971; Ney and Ortmanns, 1999; De Wachter et al., 2007). In these applications, speech is represented using discriminatively trained features to ensure that the used distance/divergence metric mostly yields lower scores for the matching class compared to the other classes, resulting in increased recognition accuracies. The input speech segments can be simply classified as the label of the closest exemplar, or by a voting scheme on the set of K nearest neighbors (Golipour and O’Shaughnessy, 2009).

Exemplar-based sparse representations (SR) is an alternative data-driven ASR approach in which the spectrogram of input speech segments is modeled as a sparse linear combination of exemplars. SR-based techniques have been successfully used for speech enhancement (Gemmeke et al., 2011b), feature extraction (Sainath et al., 2010) and speech recognition (Kanevsky et al., 2010; Hurmalainen et al., 2011; Gemmeke et al., 2011a; Tan and Narayanan, 2012). These approaches model the acoustics using same-length exemplars labeled on the frame level and stored in a single overcomplete dictionary. The exemplar weights are obtained by solving a regularized convex optimization problem with a cost function consisting of the approximation quality with respect to a divergence and a term to induce sparse linear combinations using only a few exemplars. The choice of the divergence depends on the used speech fea- 
tures (how speech and noise sources are distributed in the highdimensional feature space) to obtain reasonable sparse linear combinations. The non-negativity requirement of the SR formulation prevents the use of discriminatively trained features in this framework. The generalized Kullback-Leibler divergence (KLD) with the mel scaled magnitude spectral features has been successfully used in various applications in source separation, SR-based noise robust speech recognition and polyphonic music transcription (Virtanen, 2007; Smaragdis and Brown, 2003; Smaragdis, 2007; Raj et al., 2010; Tan and Narayanan, 2012). King et al. investigated the optimal parameter of the beta divergence as a cost function for non-negative matrix factorizationbased speech separation and music interpolation in (King et al., 2012).

This paper focuses on the divergence used by a recently proposed exemplar matching-based recognition approach, dubbed noise robust exemplar matching (N-REM) (Y1lmaz et al., 2014a), which performs conventional exemplar matching in a SR formulation to be able to model noisy speech. Similar to the exemplar matching approaches, N-REM uses exemplars associated with a single speech unit such as phones, syllables, half-words or words. These exemplars are organized in separate dictionaries based on their duration (frame length) and class (associated speech unit). By applying a sliding window approach, the noisy speech segments are jointly approximated as a linear combination of the speech and noise exemplars using each dictionary. The recognizer adopts a reconstruction error based back-end, i.e. the recognition is performed by comparing the approximation quality for different classes quantified by a divergence measure and choosing the class sequence that minimizes the total reconstruction error.

The divergence plays an essential role in the recognition performance of N-REM on account of the reconstruction error based backend. The optimal divergence is expected to weight the individual reconstruction errors of each time-frequency cells in a way that the most informative cells contribute the most to the total reconstruction error. In this work, we use the Alpha-Beta (AB) divergence (Cichocki et al., 2011) in place of the generalized KLD to quantify the approximation error. The AB divergence is a family of divergences with two parameters, namely $\alpha$ and $\beta$. For different values of these parameters, the $\mathrm{AB}$ divergence connects various well-known distance/divergence measures such as the squared Euclidean distance, Hellinger distance, Itakura-Saito divergence and generalized KLD. The higher degree of freedom offered by the AB divergence has been shown to enable better robustness against noise and outliers (Cichocki et al., 2011).

The main contribution of this paper is a novel noise robust recognizer which traverses the $(\alpha, \beta)$ plane based on the estimated SNR level to perform the most accurate separation of speech and noise sources. The recognition performance of the proposed system is investigated on the small vocabulary track of the $2^{\text {nd }}$ CHiME Challenge (CHIME-2) and the AURORA-2 database. The initial ASR results at lower SNR levels (-6 dB and $0 \mathrm{~dB}$ of the CHIME-2 data) for numerous $(\alpha, \beta)$ pairs are presented in (Y1lmaz et al., 2014b) and it has been shown that using $\mathrm{AB}$ divergence with an appropriate $(\alpha, \beta)$ pair provides better recognition than the generalized KLD with tuned sparsity. In this work, we extend the recognition experiments to all SNR levels of both databases to have a better understanding of the novel system using the $\mathrm{AB}$ divergence. The baseline system which uses the generalized KLD as a dissimilarity measure is described in (Y1lmaz et al., 2014a). Secondly, an indepth discussion on the impact of the divergence parameters on the recognition performance is provided by comparing the behavior of the generalized KLD and AB divergence for several $(\alpha, \beta)$ pairs. Finally, we apply the adaptive noise modeling technique, active noise exemplar selection (ANES) (Y1lmaz et al., 2014a), on the CHIME-2 data to investigate the recognition performance in case of genuine room noise. The rest of the paper is organized as follows. The N-REM using the AB divergence is described in Section 2. Section 3 discusses the evaluation setup and implementation details. Section 4 presents the recognition results and a discussion about the results is given in Section 5. Section 6 provides a general discussion and the concluding remarks.

\section{Noise Robust Exemplar Matching}

N-REM models noisy speech segments as a sparse linear combination of speech and noise exemplars of various lengths that are stored in multiple dictionaries. The overview of the recognizer is given in Figure 1. Compared to a system using fixedlength exemplars stored in a single dictionary, using separate dictionaries for each class results in noisy speech segments being approximated as a linear combination of exemplars belonging to the same class only. From the geometrical interpretation of SR-based source separation, it is known that the farther the convex hull of the basis vectors belonging to different sources (speech and noise in this case) are, the better the separation is (Donoho and Stodden, 2004). Hence, the use of separate dictionaries for each speech unit provides a more precise representation in the high-dimensional feature space.

\subsection{Model Description}

Training frame sequences representing various speech units (speech exemplars) are extracted based on the state-level alignments obtained using a conventional HMM-based recognizer. Speech exemplars, each comprised of $D$ mel frequency bands and spanning $l$ frames, are reshaped into a single vector and stored in the columns of a speech dictionary $\mathbf{S}_{c, l}$ : one for each class $c$ and each frame length $l$. Each dictionary is of dimensionality $D l \times N_{c, l}$ where $N_{c, l}$ is the number of available speech exemplars of class $c$ and frame length $l$. Similarly, a noise dictionary $\mathbf{N}_{l}$ for each frame length $l$ is formed by reshaping the noise exemplars. Each speech dictionary is concatenated with the noise dictionary of the same length to form a combined dictionary $\mathbf{A}_{c, l}=\left[\mathbf{S}_{c, l} \mathbf{N}_{l}\right]$ of dimensionality $D l \times M_{c, l}$ where $M_{c, l}$ is the total number of available speech and noise exemplars.

An observed noisy (and/or reverberated) speech segment of frame length $T$ frames is also reshaped into vectors by applying a sliding window approach (Gemmeke et al., 2011a) with window length of $l$ frames and stored in an observation matrix 


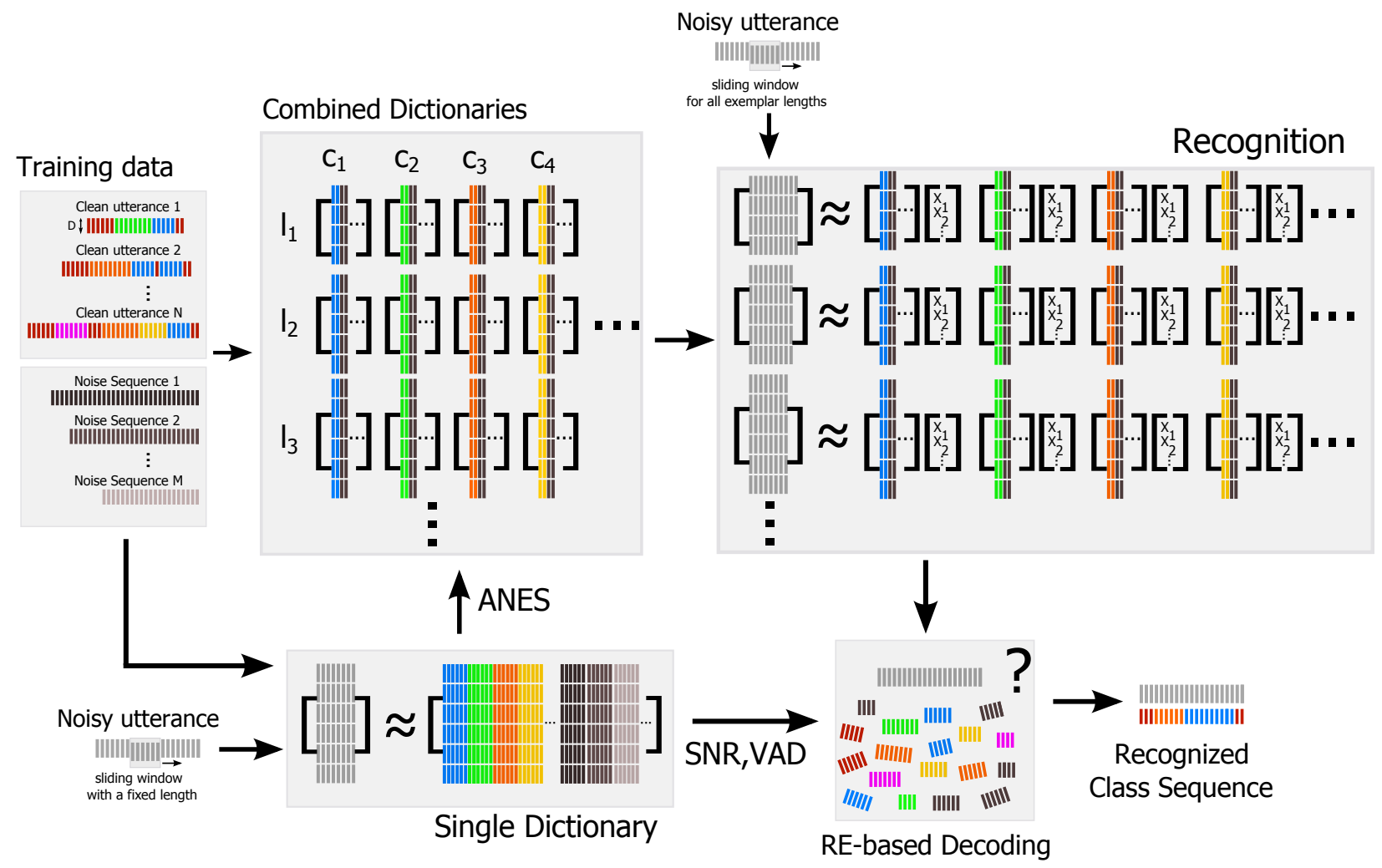

Figure 1: The Recognizer Overview. The single dictionary is used for the VAD, SNR estimation and active noise exemplar selection (ANES). Noise exemplars that are used in the recognition are selected based on the single dictionary. Speech exemplars are extracted from the training data using the segmentation information. They are organized in dictionaries based on their length and class. Noise dictionaries are concatenated to the speech dictionaries forming the combined dictionaries. Non-negative sparse coding (NSC) is applied to approximate noisy test utterances using the combined dictionaries. After a fixed number of iterations, the reconstruction errors are calculated and a dynamic programming algorithm is applied to find the class sequence with the minimum reconstruction error.

$\mathbf{Y}_{l}=\left[\mathbf{y}_{l}^{1}, \mathbf{y}_{l}^{2} \ldots, \mathbf{y}_{l}^{(T-l+1)}\right]$ of dimensionality $D l \times(T-l+1)$. Due to multiple-length exemplars, the window length $l$ is varied between the minimum exemplar length $l_{\min }$ and maximum exemplar length $l_{\max }$ yielding observation matrices $\mathbf{Y}_{l}$ for $l_{\min } \leq l \leq$ $l_{\max }$. For every class $c$, each observation vector $\mathbf{y}_{l}$ is expressed as a linear combination of the exemplars that are stored in the dictionaries of the same length:

$$
\mathbf{y}_{l} \approx \sum_{m=1}^{M_{c, l}} x_{c, l}^{m} \mathbf{a}_{c, l}^{m}=\mathbf{A}_{c, l} \mathbf{x}_{c, l} \quad \text { s.t. } \quad x_{c, l}^{m} \geq 0
$$

where $\mathbf{x}_{c, l}$ is an $M_{c, l}$-dimensional non-negative weight vector. The sparse solutions of $\mathbf{x}_{c, l}$ yield a more realistic approximation of the observed segments without overfitting and have been shown to provide better recognition results (Hoyer, 2004; Virtanen, 2007). The combined dictionaries consisting of speech and noise exemplars are presumed to model all acoustic variability in the observed signal due to pronunciation variation, background noise and so forth. This model can also model reverberation by storing reverberated speech exemplars rather than clean speech exemplars.

\subsection{Finding Exemplar Weights}

The exemplar weights $\mathbf{x}_{c, l}$ are obtained by minimizing the cost function consisting of a single term which quantifies the approximation error $d\left(\mathbf{y}_{l}, \mathbf{A}_{c, l} \mathbf{x}_{c, l}\right)$ for non-negative exemplar weights. This optimization problem can be solved with the nonnegative sparse coding (NSC) (Lee and Seung, 2000; Hoyer, 2002). Unlike the baseline recognizer and the other SR-based approaches, the new cost function does not enforce sparsity on the exemplar weights. The impact of the missing sparsity inducing term is investigated in Section 4.1 .1 by visualizing the sparseness of the obtained exemplar weights that are obtained by only minimizing the approximation error.

The value of the approximation error is highly dependent on the divergence measure $d$ and the representation of speech and noise sources. Particularly, the adopted divergence measure is expected to provide more reliable reconstruction errors by emphasizing the reliable and informative time-frequency bins which are dominated by the desired source (speech in this case). Prior work has shown that the mel-scaled spectral features provide better source separation when used in conjunction with the generalized KLD compared to the Euclidean distance (Virtanen, 2007).

Recently, the AB divergence has been proposed and its applications as a cost function for non-negative matrix factorization have been investigated (Cichocki et al., 2011). Motivated by its capabilities to weight and scale the individual ratios of the noisy speech and its approximation, $\mathbf{y}_{l}^{i} / \hat{\mathbf{y}}_{c, l}^{i}$ where $\hat{\mathbf{y}}_{c, l}=\mathbf{A}_{c, l} \mathbf{x}_{c, l}$, we investigate the recognition performance of the proposed system using the $\mathrm{AB}$ divergence for $d$. The influence of different $(\alpha, \beta)$ values on this ratio is detailed in (Cichocki et al., 2011). 
The $\mathrm{AB}$ divergence is defined as

$$
d_{A B}^{(\alpha, \beta)}(\mathbf{y}, \hat{\mathbf{y}})= \begin{cases}-\frac{1}{\alpha \beta} \sum_{k=1}^{K}\left(y_{k}^{\alpha} \hat{y}_{k}^{\beta}-\frac{\alpha}{\gamma} y_{k}^{\gamma}-\frac{\beta}{\gamma} \hat{y}_{k}^{\gamma}\right) & \text { for } \alpha, \beta, \gamma \neq 0, \\ \frac{1}{\alpha^{2}} \sum_{k=1}^{K}\left(y_{k}^{\alpha} \log \left(\frac{y_{k}^{\alpha}}{\hat{y}_{k}^{\alpha}}\right)-y_{k}^{\alpha}+\hat{y}_{k}^{\alpha}\right) & \text { for } \alpha \neq 0, \beta=0 \\ \frac{1}{\alpha^{2}} \sum_{k=1}^{K}\left(\log \left(\frac{\hat{y}_{k}^{\alpha}}{y_{k}^{\alpha}}\right)+\frac{y_{k}^{\alpha}}{\hat{y}_{k}^{\alpha}}-1\right) & \text { for } \alpha=-\beta \neq 0, \\ \frac{1}{\beta^{2}} \sum_{k=1}^{K}\left(\hat{y}_{k}^{\beta} \log \left(\frac{\hat{y}_{k}^{\beta}}{y_{k}^{\beta}}\right)-\hat{y}_{k}^{\beta}+y_{k}^{\beta}\right) & \text { for } \alpha=0, \beta \neq 0, \\ \frac{1}{2} \sum_{k=1}^{K}\left(\log \left(y_{k}\right)-\log \left(\hat{y}_{k}\right)\right)^{2} & \text { for } \alpha, \beta=0\end{cases}
$$

where $\gamma=\alpha+\beta$. The two parameters of the AB divergence can be automatically adjusted based on the amount of contamination in the target utterance as the recognition performance for different noise levels depends on the emphasized (reliable) time-frequency bins. For the NSC solution, we apply the relaxation and non-linear projection techniques proposed in $(\mathrm{Ci}-$ chocki et al., 2006) for faster convergence to the multiplicative update rule derived in (Cichocki et al., 2011) to minimize the approximation error. The multiplicative update rule which minimizes the approximation error using the $\mathrm{AB}$ divergence for $\alpha \neq 0$ is given by

$$
\mathbf{x}_{c, l} \leftarrow\left(\mathbf{x}_{c, l} \odot\left(\left(\mathbf{A}_{c, l}^{T} \mathbf{Z}_{c, l}\right) \oslash\left(\mathbf{A}_{c, l}^{T}\left(\mathbf{A}_{c, l} \mathbf{x}_{c, l}\right)^{\cdot[\gamma-1]}\right)\right)^{\cdot[\omega / \alpha]}\right)^{\cdot[1+\theta]}
$$

where $\mathbf{Z}_{c, l}=\mathbf{y}_{l}^{[\alpha]} \odot\left(\mathbf{A}_{c, l} \mathbf{x}_{c, l}\right)^{[\beta-1]}$ and ${ }^{\left[{ }^{[}\right]}$denotes element-wise exponentiation. $\omega$ is a value between $(0,2)$ and $\theta$ is a very small positive number (Cichocki et al., 2006).

\subsection{Decoding}

All observation matrices $Y_{l}$ for $l_{\min } \leq l \leq l_{\max }$ are approximated using the combined dictionaries $\mathbf{A}_{c, l}$ of the corresponding length by applying the multiplicative update rule in Equation (3). To quantify the approximation quality, we use the reconstruction error between the noisy speech segments and their approximations. The multiplicative update rule is applied iteratively until the reconstruction error provides enough discrimination between different classes. The number of iterations that satisfies this criterion has been investigated in pilot experiments. After a fixed number of iterations for all dictionaries, the reconstruction errors between the observation matrix $\mathbf{Y}_{l}$ and its approximations $\mathbf{A}_{c, l} \mathbf{x}_{c, l}$ are calculated for $l_{\min } \leq l \leq l_{\max }$. As the label of each dictionary is known, decoding is performed by applying a single-stage dynamic programming algorithm (Ney, 1984) to find the class sequence that minimizes the reconstruction error (taking the grammar into account if necessary). This search problem is visualized as a three-dimensional grid search over grid points $(x, y, z)$ which are defined by the time frames $x$ of a noisy speech segment, time frames $y$ of its approximation and the dictionary number $z$ (Ney, 1984). Noisy speech segments are only matched with the dictionaries of the same duration, i.e. no time warping is performed.

\subsection{Preprocessing of Noisy Speech}

Before the recognition phase, the noisy speech is approximated using a single overcomplete dictionary to gather some information about the target utterance such as the voice activity detection (VAD), signal-to-noise ratio (SNR) estimation and noise characteristics. This single dictionary is formed by choosing an exemplar length $L_{s}$ containing a vast number of samples from each class. The single speech dictionary $\mathbf{S}_{L_{s}}^{*}$ contains speech exemplars of all classes with the same length. The single noise dictionary $\mathbf{N}_{L_{s}}^{*}$ has noise exemplars that are extracted from the noise-only training sequences. The preprocessing step performs non-negative sparse coding using the single (combined) dictionary $\mathbf{A}_{L_{s}}^{*}=\left[\mathbf{S}_{L_{s}}^{*} \mathbf{N}_{L_{s}}^{*}\right]$

$$
\mathbf{Y}_{L_{s}} \approx \mathbf{A}_{L_{s}}^{*} \mathbf{x}_{L_{s}} \quad \text { s.t. } \quad \mathbf{x}_{L_{s}} \geq 0 .
$$

where $\mathbf{Y}_{L_{s}}$ is the observation matrix having a window length of $L_{s}$ frames. As the proposed recognizer uses an SNR-dependent $(\alpha, \beta)$ pair, the generalized KLD is used as a reference for obtaining the weights of exemplars in the single dictionary. The multiplicative update rule for finding the exemplar weights $\mathbf{x}_{L_{s}}$ is given as

$\mathbf{x}_{L_{s}} \leftarrow\left(\mathbf{x}_{L_{s}} \odot\left(\left(\left(\mathbf{A}_{L_{s}}^{*}\right)^{T}\left(\mathbf{Y}_{L_{s}} \oslash\left(\mathbf{A}_{L_{s}}^{*} \mathbf{x}_{L_{s}}\right)\right)\right) \oslash\left(\left(\mathbf{A}_{L_{s}}^{*}\right)^{T} \mathbf{1}+\mathbf{\Lambda}\right)\right)^{\left[{ }^{[\omega]}\right.}\right)^{.[1+\theta]}$

where $\mathbf{1}$ is a $\left(D \cdot L_{s}\right)$-dimensional vector with all elements equal to unity.

The information provided by the exemplar weights $\mathbf{x}_{L_{s}}$ are used for multiple purposes. Firstly, a known problem of SR approaches working on magnitude spectra is that the silence exemplars are hard to recognize: perfect silence is modeled with zero weights of all exemplars (Gemmeke et al., 2011a). In a practical noisy mixture, it is well-approximated by combining speech and noise exemplars with small weights, thus all classes will score equally well. To overcome this problem, the reconstruction errors belonging to the silence dictionaries have to be compensated for the noisy speech segments which do not contain speech. For this purpose, the recognizer embodies the preprocessing step to perform VAD for predicting whether a noisy speech segment contains speech and to estimate the SNR level for adjusting the amount of compensation. An indicator of the SNR level, $\mathrm{SNR}_{\text {ind }}$, is calculated as the ratio of total speech weights and total speech and noise weights is used in order to limit the range to $[0,1]$,

$$
\mathrm{SNR}_{\text {ind }}=\frac{\sum_{w=1}^{W} \sum_{m=1}^{J} x_{L_{s}}^{w, m}}{\sum_{w=1}^{W} \sum_{m=1}^{M} x_{L_{s}}^{w, m}} .
$$

$\mathbf{x}_{L_{s}}^{w}$ is the sparse weight vector corresponding to $w^{\text {th }}$ of $W$ noisy segments of length $L_{s} . J$ is the number of speech exemplars and $M$ is number of all exemplars.

The preprocessing step also provides useful information about the spectrotemporal content of the background noise. This information is used for extracting a small set of noise exemplars that are able to model the actual noise conditions by applying the active noise exemplar selection (ANES) technique. The level of the background noise is quantified by $\mathrm{SNR}_{\text {ind }}$ and the number of noise exemplars that are used in the recognition phase is chosen based on this value. In practice, the recognizer 
uses more noise exemplars for lower SNR levels and less or no exemplars for higher SNR levels. This way of noise modeling has been shown to both reduce the computational complexity and improve the recognition accuracies at higher SNRs. The adaptive and SNR-dependent noise modeling approach are described in Section 2.5.

Finally, the proposed recognizer chooses the divergence parameters $(\alpha, \beta)$ according to the $\mathrm{SNR}_{\text {ind }}$ value to provide better separation and improve the recognition performance. The path providing the best recognition performance on the $(\alpha, \beta)$ plane is determined in advance on development data and the divergence parameters are chosen on this predetermined path based on the estimated SNR level.

\subsection{Speech and Noise Dictionaries}

Several dictionary design techniques have been applied for effective speech and noise modeling using the exemplars. As the speech exemplars are associated with a single speech unit, their length distribution is class-dependent which results in unevenly populated speech dictionaries. Speech dictionary design mainly involves increasing the number of exemplars in underpopulated speech dictionaries to avoid poor acoustic modeling. Prewarping (Yilmaz et al., 2013b) is applied to increase the number of the exemplars by removing a small number of frames, excluding the very first and last frame, from an exemplar of length $l$ to obtain shorter exemplars of length $l_{\text {new }}<l$.

Noise exemplars are extracted from noise-only training sequences for arbitrary length. While there are a vast number of noise exemplars for every exemplar length, only the ones that match the actual noise conditions will be useful during the recognition. As a result, noise dictionary design mainly focuses on accurate modeling of the background noise using the smallest possible number of noise exemplars. ANES is an adaptive way of noise modeling that accurately picks a small number of noise exemplars that can model the actual noise conditions. Large performance gains have been reported compared to fixed noise modeling, i.e. using the same set of noise exemplars for all test utterances, especially at lower SNRs (Y1lmaz et al., 2014a). Adaptive noise dictionaries are obtained based on the noise weights that are provided by the single dictionary setup described in Section 2.4. This technique aims to select a small number of noise exemplars that can accurately model the actual noise conditions. An equal number of exemplars is extracted from a large number of noise-only training sequences and stacked in a single noise dictionary. In order to identify which noise-only training sequences can accurately model the actual noise conditions, all weights belonging to the noise exemplars extracted from each noise-only training sequence are accumulated. With the same motivation as discussed in (Y1lmaz et al., 2013a), noise exemplars used for the recognition are extracted from the most active noise-only training sequences, i.e. the sequences with the highest weights.

Another technique that has been proposed for improved noise modeling in SR-based recognition systems is called noise sniffing (Gemmeke and Virtanen, 2010). This technique acquires noise exemplars on the fly from the immediate neighborhood of the target utterance. The extracted noise exemplars are added to the combined dictionaries and used for the recognition. In case of limited noise context, a small number of frames from the beginning and end of the target utterance are extracted and contained in combined dictionaries. Shifted copies of these frame sequences are also included to provide some degree of shiftinvariance (Gemmeke and Van hamme, 2012). The VAD information is used to detect the speech onset and offset points.

SNR-dependent noise modeling approach finds a compromise between the accuracy of the noise modeling and computational complexity by adjusting the amount of the noise exemplars in the combined dictionaries depending on the estimated SNR level. At lower SNRs, a larger number of noise-only training sequences are used for noise exemplar extraction. Consequently, computational complexity of the recognizer is reduced at high SNRs without loss of recognition accuracy while preserving the noise modeling capabilities at lower SNRs. Moreover, SNR-dependent noise modeling provides gains in the recognition accuracy of clean speech, as the dictionaries contain only a few noise exemplars during the recognition of clean speech.

\section{Experimental Setup}

\subsection{Databases}

\subsubsection{AURORA-2}

The recognition performance of N-REM is first evaluated on the test set A and B of the AURORA-2 corpus (Hirsch and Pearce, 2000). The training material of AURORA-2 consists of a clean and a multi-condition training set, each containing 8440 utterances with one to seven digits in American English. The multi-condition training set was constructed by mixing the clean utterances with noise at SNR levels of 20, 15, 10 and 5 $\mathrm{dB}$.

Test set A consists of 4 clean and 24 noisy datasets with four noise types (subway, babble, car and exhibition) at six SNR levels, 20, 15, 10, 5, 0 and $-5 \mathrm{~dB}$. The noise types of this test set match the multi-condition training set. Test set $\mathrm{B}$ has the same number of test sets with four different noise types (restaurant, street, airport, station) at the same SNR levels. Each subset contains 1001 utterances. To reduce the simulation times, we subsampled the test sets by a factor of 4 (250 utterances per test set, 1000 utterances per SNR). A different subset with 100 utterances from each test set is used for development purposes. All data has a sampling frequency of $8 \mathrm{kHz}$.

\subsubsection{CHIME-2}

The small vocabulary track of the $2^{\text {nd }}$ CHiME Challenge (Vincent et al., 2013) addresses the problem of recognizing commands in a noisy living room. The clean utterances in the CHIME-2 data are taken from the GRID corpus (Cooke et al., 2006) which contains utterances from 34 speakers reading 6word sequences of the form command-color-preposition-letterdigit-adverb. There are 25 different letters, 10 different digits and 4 different alternatives for each of the other classes. Even though there is no silence between the words, leading silences 


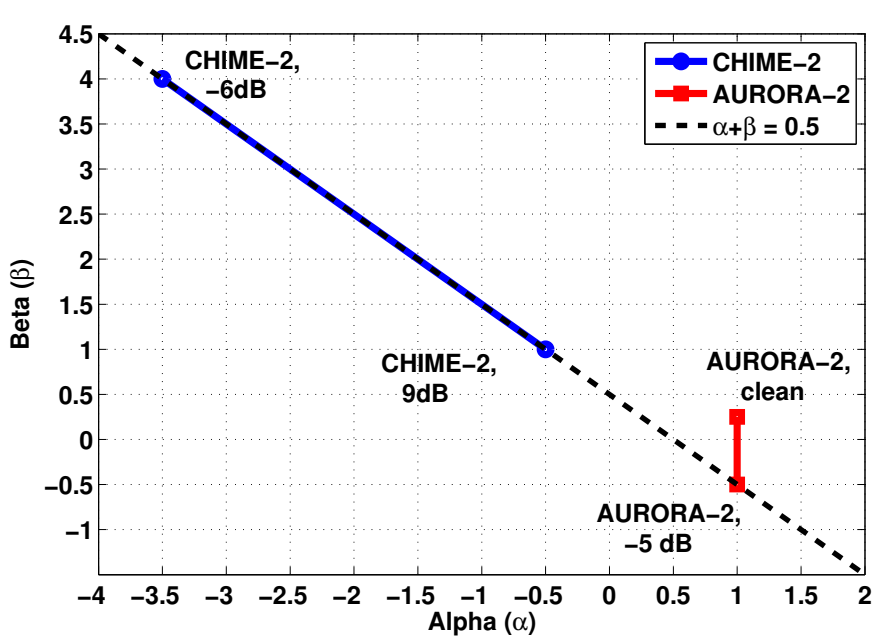

Figure 2: The line segments on the $\mathrm{AB}$ plane used for the recognition of the AURORA-2 and CHIME- 2 databases - The $\alpha+\beta=0.5$ line is also visualized which provided the best results for noisy conditions on both databases

of variable duration exist occasionally. The recognition accuracy of a system is calculated based on the correctly recognized letter and digit keywords.

The clean utterances are convolved with binaural room impulse responses with speaker head movement effects which are recorded in a living room. Then, the resulting reverberated utterances are mixed with binaural recordings of genuine room noise recorded in the same living room at SNR levels of 9, 6, $3,0,-3$ and $-6 \mathrm{~dB}$. The training set contains 500 utterances per speaker $(17,000$ utterances in total) with clean, reverberated and noisy versions. Noisy utterances are provided both in isolated or embedded form. Embedded recordings contain 5 seconds of background noise before and after the target utterance. The development and test sets contain 600 utterances from all speakers at each SNR level (3600 utterances in total for each set) both in isolated and embedded from. The immediate noise context of the target utterances are available in 164 embedded recordings in the development set and 176 embedded recordings in the test set. All data has a sampling frequency of $16 \mathrm{kHz}$.

\subsection{Dictionary Creation and Implementation Details}

\subsubsection{AURORA-2}

The speech exemplars are extracted from the clean training set. Acoustic feature vectors are represented in mel-scaled magnitude spectra with 23 frequency bands. The speech exemplars representing half-digits are segmented by a conventional HMM-based system. The recognizer uses in total 52295 speech exemplars excluding 990 silence exemplars. The number of noise exemplars varies depending on the duration of the noise-only sequences that are selected by ANES. On average, the recognizer uses 11355 and 1044 noise exemplars/utterance in total at SNR level of $-5 \mathrm{~dB}$ and clean speech respectively. The minimum and maximum exemplar lengths are 8 and 40 frames respectively. Exemplars longer than 40 frames are omitted to limit the number of dictionaries. The recognizer uses in total 675 dictionaries of 23 different classes (half-digits plus silence). The combined dictionaries and observation matrices are $l_{2}$-normalized for all SNR levels. The multiplicative update rule is iterated 100 times for convergence of all frame lengths with $\omega=1.75$ and $\theta=0.008$. The divergence parameters $(\alpha, \beta)$ providing the best performance at the lowest and highest SNR are investigated on the development data. The pilot experiments on the development data have shown that the best results are obtained on the line $\alpha=1$ for the AURORA-2 database. The AB divergence with $(1,-0.5)$ and $(1,0.25)$ provided the best recognition accuracies at SNR level of $-5 \mathrm{~dB}$ and on clean speech. The line segments used during the recognition of both databases are illustrated in Figure 2 on the $\mathrm{AB}$ plane. For the recognition of AURORA-2, the $\beta$ value is estimated in the interval of $[-0.5,0.25]$ as a linear function of the $\mathrm{SNR}_{\text {ind }}$ value,

$$
\left.\beta=\max \left(\min \left(2 \cdot \mathrm{SNR}_{\mathrm{ind}}-0.55\right), 0.25\right),-0.5\right) .
$$

The single noise dictionary contains noise exemplars that are extracted from 800 longest noise-only training sequences (50 sequences from each multicondition training set). From each noise-only training sequence, 10 noise exemplars are extracted with equal frame shifts resulting in 8000 noise exemplars. The single speech dictionary contains 2200 speech exemplars (100 exemplars from each class excluding silence). The speech and noise exemplars contain 15 frames. The first and last 20 frames of the target utterances are assumed not to contain speech and 150 noise exemplars with 15 frames ( 5 exemplars and 70 shifted copies from each end) are extracted and concatenated to the single dictionary. The speech and noise exemplar weights are obtained after 300 iterations.

The silence compensation is performed after obtaining the weights by applying the multiplicative update rule in Equation (5). The speech component is reconstructed by linearly combining the speech exemplars only and a frame-level estimation of the speech activity (FSA) is obtained by summing over the frequency bins of the reconstructed speech. FSA is normalized to contain values between $[0,1]$ over the complete utterance and inverted such that 1 denotes the silence and 0 denotes the maximum observed speech activity. Then, in order to obtain steeper transitions between speech and silence regions, we calculate the speech activity value (VAD) by applying a shifted and scaled logistic function Gemmeke et al. (2011a) to the FSA values

$$
\mathrm{VAD}=\frac{1}{1+\exp \left(c_{1} * \mathrm{FSA}-\eta\right)}
$$

with the parameters $c_{1}$ and $\eta . c_{1}$ is a scalar and set to $10 . \eta$ is an SNR-dependent value which is calculated as

$$
\begin{aligned}
& \eta=\frac{1-\exp \left(c_{1} * \zeta\right)}{\exp \left(c_{1} * \zeta\right)-\exp \left(c_{1}\right)} \\
& \left.\zeta=\min \left(\mathrm{SNR}_{\mathrm{ind}} * c_{2}, c_{3}\right)\right)
\end{aligned}
$$

where $c_{2}$ and $c_{3}$ are set to 1.5 and 0.55 respectively. Thresholding is applied to the VAD values to obtain a binary decision for each frame

$$
\mathrm{VAD}_{\mathrm{h}}= \begin{cases}1 & \text { if } \mathrm{VAD}>\mathrm{VAD}_{\mathrm{thr}} \\ 0 & \text { if } \mathrm{VAD}<=\mathrm{VAD}_{\mathrm{thr}}\end{cases}
$$


$\mathrm{VAD}_{\text {thr }}$ is set to 0.95 . The reconstruction errors corresponding to the silence dictionaries are scaled by a value $\mathrm{CF}$ which depends on the $\mathrm{VAD}_{\mathrm{h}}$ value assigned to the middle frame of the corresponding noisy segment and the SNR estimate,

$$
\mathrm{CF}=1-\min \left(\max \left(\mathrm{SNR}_{\mathrm{ind}} \cdot \theta, \phi\right), \gamma\right) \cdot \mathrm{VAD}_{\mathrm{h}}
$$

where $\theta$ is a scale factor, $\phi$ and $\gamma$ are lower and upper limits. They are set to $0.75,0.1$ and 0.55 respectively.

In the recognition phase, noise dictionaries are created by performing active noise exemplar selection and noise sniffing. Once the most active training sequences are found using the single dictionary, i.e. the noise sequences obtaining the highest weights, noise dictionaries that are used in the recognition phase are extracted from the $N_{\max }$ most active training sequences with hops of 3 frames (between 77-170 exemplars from each sequence). The value of $N_{\max }$ depends on the SNR level and it is chosen according to the $\mathrm{SNR}_{\text {ind }}$ value given in Equation (6):

$$
N_{\text {max }}= \begin{cases}3 & \text { if } \mathrm{SNR}_{\text {ind }}<0.35 \\ 2 & \text { if } \mathrm{SNR}_{\text {ind }}>=0.35 \text { and } \mathrm{SNR}_{\text {ind }}<0.5 \\ 1 & \text { if } \mathrm{SNR}_{\text {ind }}>=0.5 \text { and } \mathrm{SNR}_{\text {ind }}<0.65 \\ 0 & \text { if } \mathrm{SNR}_{\text {ind }}>=0.65 .\end{cases}
$$

The combined dictionaries of all classes and lengths contain the noise exemplars that are extracted from these $N_{\max }$ training sequences. The combined dictionaries contain only a few or no noise exemplars during the recognition of high SNR levels.

\subsection{2. $C H I M E-2$}

The exemplars and noisy speech segments are represented as mel-scaled magnitude spectral features extracted with a 26 channel mel-scaled filter bank $(D=26)$. The frame length is 25 $\mathrm{ms}$ and the frame shift is $10 \mathrm{~ms}$. The binaural data is averaged in the spectral domain to obtain 26-dimensional feature vectors. The exemplars are extracted from the reverberated utterances in the training set according to the state-based segmentations obtained using the acoustic models in the toolkit provided with the database. Exemplars belonging to each speaker are organized in separate dictionary sets for speaker-dependent modeling yielding 34 different dictionary sets. Based on the availability of the exemplars, the minimum and maximum exemplar lengths are 4 and 40 frames respectively.

Half-word exemplars seemed to generalize sufficiently to unseen data. Half-word exemplars are extracted by cutting the word exemplars at the HMM state yielding the minimum average length difference between the two halves. Dictionary sizes vary with different classes and speakers. Prewarping is applied to boost the modeling capabilities of the underpopulated speech dictionaries (especially for the ones belonging to letters due to the high number of alternatives and hence the small number of exemplars per class) and it is limited to a single frame. The number of exemplars in each dictionary after prewarping (Y1lmaz et al., 2013b) is limited to 50.

Each embedded utterance in the development and test set is segmented into noise-only sequences by removing all target utterances. 5 noise exemplars of 25 frames are extracted from

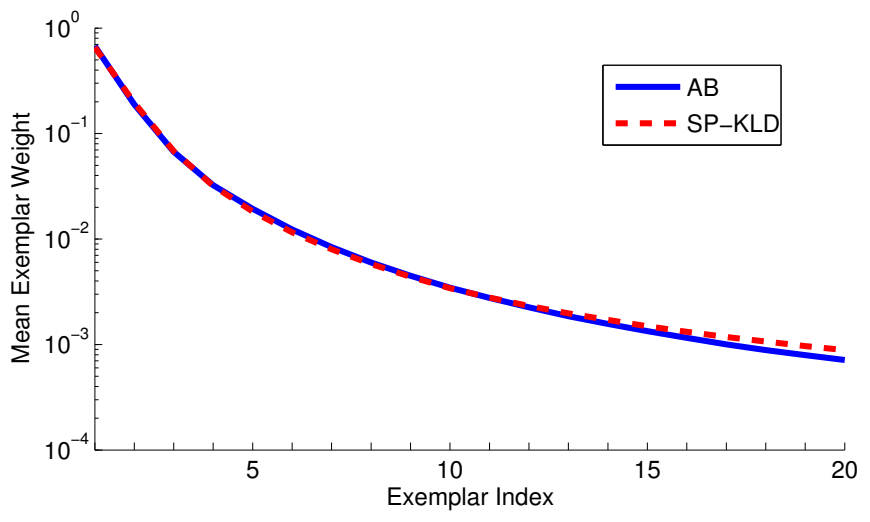

Figure 3: The comparison of average exemplar weights obtained using the generalized KLD with tuned sparsity and the AB divergence - The weights are obtained using 400 utterances at SNR level of $-5 \mathrm{~dB}$ in test set A of AURORA-

each noise-only sequence and stored in the single noise dictionary. The size of the single noise dictionary varies depending on the number of available noise-only sequences for each embedded recording. ANES only evaluates the noise-only sequences that are extracted from the embedded recording which contains the target utterance. The single speech dictionary contains 2354 full-word exemplars (maximum 50 exemplars from 51 classes) of 25 frames. The full-word exemplars are used in the single speech dictionary, as there is no exemplar length $L_{s}$ containing a vast number of samples from each half-word class.

The noise dictionaries used for the recognition phase contain 200 noise exemplars that are acquired on the fly from the immediate neighborhood of the target utterance in both directions until the frames belonging to other target utterances. In addition to these sniffed noise exemplars, 200-300 noise exemplars are extracted from the most active 2 noise-only sequences selected by ANES. These noise exemplars are extracted with jumps of 3 frames yielding a different number of noise exemplars depending on the length of the noise-only sequence. The multiplicative update rule is iterated 25 times to obtain the exemplar weights. $\omega$ and $\theta$ are set to 1.75 and 0.008 respectively. The columns of the combined dictionaries and observation matrices are $l_{2}$-normalized. To investigate the impact of the divergence parameters, we have performed recognition experiments on the lowest and highest SNR levels of the development data. The best results at $-6 \mathrm{~dB}$ and $9 \mathrm{~dB}$ are obtained using $\mathrm{AB}$ divergence with $(-3.5,4)$ and $(-0.5,1)$ respectively. Considering the results reported in (Y1lmaz et al., 2014b), the divergence parameters are chosen on the line $\alpha+\beta=0.5$ in the interval of $([-3.5,-0.5],[4,1])$ as a linear function of the $\mathrm{SNR}_{\text {ind }}$ value,

$$
\begin{gathered}
\left.\alpha=\max \left(\min \left(7.5 \cdot \mathrm{SNR}_{\text {ind }}-5.75\right),-0.5\right),-3.5\right), \\
\left.\beta=\max \left(\min \left(-7.5 \cdot \mathrm{SNR}_{\text {ind }}+6.25\right), 4\right), 1\right) .
\end{gathered}
$$

\subsection{Evaluation Metrics}

We have opted for the metrics which have been traditionally used for the evaluation of the databases described in Section 3.1 for comparability with the previous literature. The word error rate has been used to quantify the recognition accuracy for 


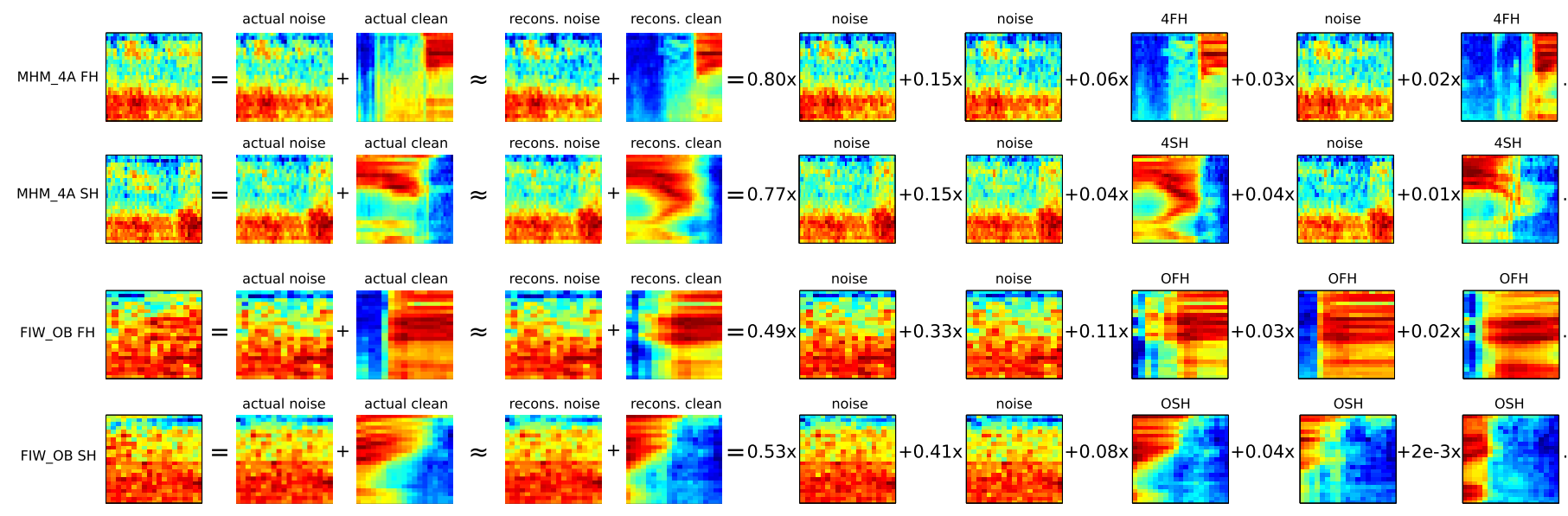

Figure 4: Illustration of the sparsity the exemplar weights provided by N-REM dictionaries using the AB divergence - The mel-scaled spectral patches given in the first column are the noisy mixtures extracted from noisy utterances MHM_4A and FIW_OB with subway and exhibition hall noise at an SNR level of -5 dB respectively. The following columns list the exemplars with the highest weights that are used to approximate the noisy segments in the first column. The label of each exemplar is given above the visual representation ('FH': first-half, 'SH': second-half)

the AURORA-2 digit recognition task. The keyword recognition accuracy is used to evaluate the system performance on the CHIME-2 data.

\section{Results}

In this section, we firstly investigate the impact of the missing sparsity inducing term in the cost function by visualizing how sparse the exemplar weights obtained using 400 noisy utterances from test set A of the AURORA-2 database and report the recognition accuracies on the same database. Then, we compare the recognition performance of N-REM using the generalized KLD with induced sparsity with and without the adaptive noise modeling technique ANES on the CHIME-2 data. Finally, the recognition accuracies provided by N-REM using the $\mathrm{AB}$ divergence on the CHIME-2 data are presented. The recognition accuracies on both databases are compared with the baseline N-REM recognizer which uses the generalized KLD with tuned sparsity and some other comparable recognition schemes such as exemplar-based sparse representations recognizers and a multicondition-trained GMM-HMM and DNN-HMM recognizer.

\subsection{AURORA-2}

\subsubsection{Sparsity of $A B$ Divergence}

The induced sparsity has been a requirement for previous SR approaches using an overcomplete dictionary to select only a few exemplars with non-zero weights among thousands. Consequently, a realistic linear approximation of noisy speech segments are obtained without overfitting. On the other hand, NREM uses dictionaries that contain a lot less exemplars than the ones used by the previous SR approaches and we investigate whether the inherent sparsity imposed due to the non-negativity constraint is enough for realistic approximations using the $\mathrm{AB}$ divergence. We perform recognition experiments at the lowest SNR level of AURORA-2 to assure that the recognizer uses the highest number of noise exemplars as discussed in Section 2.5. In Figure 3, the largest exemplar weights obtained for 400 test utterances at $-5 \mathrm{~dB}$ using the $\mathrm{AB}$ divergence are averaged and compared with the ones obtained using the generalized KLD with tuned sparsity on the same utterances. The divergence parameters are estimated based on the $\mathrm{SNR}_{\text {ind }}$ value. The sparseness of the exemplar weights is further visualized in Figure 4 by randomly picking two single-digit utterances at the same SNR level corrupted with subway and exhibition hall noise. For each noisy speech segment, the exemplars with the 5 largest weights are listed to observe how fast the weights are decaying for the dictionaries yielding the smallest reconstruction error. From these figures, it can be concluded that the linear combinations yielded by the multiplicative update rule given in Equation (3) for the best performing divergence parameters are sparse enough to realistically estimate noisy segments.

One could rightfully raise the question if a sparsity penalty to Equation (3) would be beneficial. We have not found a positive effect of doing so in pilot experiments, which is explained by the reported sparsity provided by the appropriate divergence parameters. It is important to mention that there may be other divergence parameters in which additional sparsity could help. However, this investigation requires a three-dimensional parameter search, i.e. divergence parameters and the sparsity factor, which is not considered in the scope of this work.

\subsubsection{Recognition Results}

The recognition performance of N-REM using the AB divergence is compared with the baseline N-REM using the generalized KLD with tuned sparsity (Y1lmaz et al., 2014a), a standard GMM-HMM recognizer (Gemmeke et al., 2011a) and two noise robust SR-based recognition techniques using fixedlength exemplars in a single overcomplete dictionary, namely sparse classification (SC) and feature enhancement (FE-GMMHMM) (Gemmeke et al., 2011a). The SR-based recognition techniques achieve among the best known results on AURORA2 , especially at lower SNRs, performing significantly better 
Table 1: Word error rates in percentages obtained on test set A and B of the AURORA-2 database

(a) Test set $\mathrm{A}$

\begin{tabular}{lcccccccc}
\hline SNR(dB) & clean & -5 & 0 & 5 & 10 & 15 & 20 & $0-20$ \\
\hline \hline N-REM (SP-KLD,ANES) & $\mathbf{1 . 7}$ & 19.1 & 9.2 & 5.9 & 4.9 & 3.6 & 2.4 & 5.2 \\
\hline N-REM (AB,(1,-0.5),ANES) & 2.8 & 15.5 & 10.1 & 7.8 & 6.9 & 5.6 & 4.4 & 7.0 \\
\hline N-REM (AB,(1,0.25),ANES) & 1.8 & 31.4 & 12.6 & 6.6 & $\mathbf{4 . 7}$ & $\mathbf{3 . 5}$ & $\mathbf{2 . 3}$ & 5.9 \\
\hline N-REM (AB,SNR-dep.,ANES) & 1.8 & $\mathbf{1 4 . 9}$ & $\mathbf{8 . 5}$ & $\mathbf{5 . 8}$ & $\mathbf{4 . 7}$ & $\mathbf{3 . 5}$ & $\mathbf{2 . 3}$ & $\mathbf{5 . 0}$ \\
\hline \hline GMM-HMM & 0.7 & 60.8 & 24.3 & 7.3 & 2.9 & 1.3 & 0.8 & 7.3 \\
\hline SC & 3.7 & 35.2 & 13.8 & 7.4 & 5.6 & 4.8 & 4.5 & 7.2 \\
\hline FE-GMM-HMM & 0.5 & 30.4 & 10.7 & 3.3 & 1.5 & 1.1 & 0.7 & 3.5 \\
\hline DNN-HMM & 0.5 & 52.4 & 17.9 & 3.8 & 1.5 & 0.9 & 0.7 & 5.0 \\
\hline
\end{tabular}

(b) Test set $\mathrm{B}$

\begin{tabular}{lcccccccc}
\hline SNR(dB) & clean & -5 & 0 & 5 & 10 & 15 & 20 & $0-20$ \\
\hline \hline N-REM (SP-KLD,ANES) & $\mathbf{1 . 7}$ & 55.0 & $\mathbf{2 4 . 3}$ & $\mathbf{1 0 . 1}$ & 5.5 & 3.5 & 2.7 & 9.2 \\
\hline N-REM (AB,(1,-0.5),ANES) & 2.8 & 68.0 & 35.9 & 17.1 & 8.9 & 6.8 & 6.0 & 14.9 \\
\hline N-REM (AB,(1,0.25),ANES) & 1.8 & 67.2 & 34.9 & 12.7 & 5.0 & $\mathbf{3 . 1}$ & $\mathbf{2 . 3}$ & 11.6 \\
\hline N-REM (AB,SNR-dep.,ANES) & 1.8 & $\mathbf{5 3 . 5}$ & 24.5 & 10.4 & $\mathbf{4 . 9}$ & $\mathbf{3 . 1}$ & 2.5 & $\mathbf{9 . 0}$ \\
\hline \hline GMM-HMM & 0.7 & 64.0 & 25.9 & 7.4 & 2.6 & 1.2 & 0.9 & 7.6 \\
\hline SC & 3.7 & 52.4 & 23.5 & 11.0 & 5.9 & 2.7 & 4.5 & 9.9 \\
\hline FE-GMM-HMM & 0.5 & 52.6 & 20.5 & 5.7 & 2.1 & 1.2 & 0.5 & 6.0 \\
\hline DNN-HMM & 0.5 & 62.9 & 24.3 & 6.9 & 2.0 & 1.1 & 0.5 & 6.7 \\
\hline
\end{tabular}

than for instance the ETSI advanced front-end (AFE) which has been considered as a reference for the AURORA-2 database (Hirsch and Pearce, 2006). The GMM-HMM, DNN-HMM and FE-GMM-HMM recognition systems are trained on the multicondition training set. The DNN-HMM system uses a twolayered p-norm DNN (Zhang et al., 2014) with 1000 input and 200 output dimensions trained according to (Povey et al., 2015). The input layer uses 13 mel-frequency cepstral cooefficients (MFCC) with a temporal context of \pm 7 frames. The overcomplete dictionary used by SC and FE-GMM-HMM recognizers contain 10000 speech and 5000 noise exemplars with exemplar length of 30 frames. The exemplar weights are obtained after 600 iterations. We have performed recognition experiments on the same subset containing 1000 utterances from each SNR to obtain comparable recognition results.

The word error rates (WER) obtained on the test set A and $B$ are given in Table 1. The upper panel presents the WER results provided by the baseline system and proposed recognizers using fixed divergence parameters of $(1,-0.5),(1,0.25)$ and SNR-dependent divergence parameters according to Equation (7). The best results of the baseline and proposed N-REM systems are given in bold for each SNR level. The baseline NREM provides WERs of $19.1 \%$ and $9.2 \%$ at SNR levels of -5 $\mathrm{dB}$ and $0 \mathrm{~dB}$. The proposed system with the SNR-dependent divergence parameters performs better than the baseline with WERs of $14.9 \%$ and $8.5 \%$ at the same SNR levels with an absolute improvement of $4.2 \%$ and $0.7 \%$. From these results, it can be concluded that the recognizer with the SNR-dependent parameters achieves the accurate recognition provided by the $\mathrm{N}$-REM systems using fixed divergence parameters of $(1,-0.5)$ and $(1,0.25)$ at low and high SNR levels respectively. Moreover, these WERs are substantially lower than $35.2 \%$ and $13.8 \%$ of the SC recognizer and $30.4 \%$ and $10.7 \%$ of the FE-GMMHMM recognizer.

At the higher SNR levels, using the $\mathrm{AB}$ divergence does not have a considerable impact on the performance. The average WER between $0 \mathrm{~dB}$ and $20 \mathrm{~dB}$ slightly decreases from $5.2 \%$ to 5.0\%. N-REM performs better than SC and GMM-HMM at 5 $\mathrm{dB}$ with a WER of $5.8 \%$ compared to $7.3 \%$ of GMM-HMM, $7.4 \%$ of SC and $3.8 \%$ of DNN-HMM. FE-GMM-HMM and DNN-HMM provide the best results at $5 \mathrm{~dB}$ and $10 \mathrm{~dB}$ with comparable WERs. At $15 \mathrm{~dB}$ and $20 \mathrm{~dB}$, there is a performance gap between the N-REM and SC recognizers and the GMMHMM and FE-GMM-HMM recognizers which benefit from the enhanced discriminative power of complex GMMs used in conjunction with MFCC features. N-REM performs better than SC with a WER of $3.5 \%$ at $15 \mathrm{~dB}$ and $2.3 \%$ at $20 \mathrm{~dB}$ compared to $4.8 \%$ and $4.5 \%$ of SC. The recognition performance on test set $\mathrm{B}$ is given in the lower panel of Table 1 . In general, using the $\mathrm{AB}$ divergence does not have a noticeable influence in a mismatched noise scenario.

\subsection{CHIME-2}

For the CHIME-2 data, the GMM-HMM recognizer uses speaker-dependent acoustic models trained on noisy data. These results are obtained using the HTK recognition toolkit and the details are available at the $2^{\text {nd }}$ CHIME Challenge website $^{1}$. The details of the SC, FE-GMM-HMM and HMM-FE

${ }^{1}$ http://spandh.dcs.shef.ac.uk/chime_challenge/chime2013/ chime2_task1.html 
Table 2: Keyword recognition accuracies in percentages obtained on the development and test set of the CHIME-2 database

(a) Development Set

\begin{tabular}{lccccccc}
\hline SNR(dB) & -6 & -3 & 0 & 3 & 6 & 9 & $A v g$ \\
\hline \hline N-REM (SP-KLD) & 69.4 & 76.4 & 85.0 & 90.1 & $\mathbf{9 2 . 9}$ & 93.3 & 84.5 \\
\hline N-REM (SP-KLD,ANES) & 70.4 & 77.9 & 84.8 & 90.4 & 92.6 & $\mathbf{9 3 . 8}$ & 85.0 \\
\hline N-REM (AB,(-0.5,1),ANES) & 72.9 & 78.0 & 85.9 & $\mathbf{9 1 . 2}$ & 90.8 & 93.0 & 85.3 \\
\hline N-REM (AB,(-3.5,4),ANES) & 75.2 & $\mathbf{7 8 . 9}$ & 86.1 & 90.4 & 91.2 & 92.7 & 85.7 \\
\hline N-REM (AB,SNR-dep.,ANES) & $\mathbf{7 5 . 4}$ & 78.8 & $\mathbf{8 6 . 3}$ & 90.5 & 91.2 & 92.7 & $\mathbf{8 5 . 8}$ \\
\hline \hline GMM-HMM & 49.3 & 58.7 & 67.5 & 75.1 & 78.8 & 82.9 & 68.7 \\
\hline FE-GMM-HMM & 68.0 & 72.2 & 80.9 & 86.7 & 89.0 & 90.5 & 81.2 \\
\hline HMM-FE & 69.1 & 73.6 & 81.5 & 87.3 & 89.4 & 90.3 & 81.9 \\
\hline SC & 75.5 & 81.4 & 87.5 & 89.9 & 92.4 & 92.3 & 86.5 \\
\hline
\end{tabular}

(b) Test set

\begin{tabular}{lccccccc}
\hline SNR(dB) & -6 & -3 & 0 & 3 & 6 & 9 & $A v g$ \\
\hline \hline N-REM (SP-KLD) & 69.3 & 76.8 & 84.5 & $\mathbf{8 8 . 8}$ & $\mathbf{9 1 . 9}$ & $\mathbf{9 3 . 5}$ & 84.1 \\
\hline N-REM (SP-KLD,ANES) & 71.0 & 78.9 & 85.3 & 88.7 & $\mathbf{9 1 . 9}$ & 92.8 & 84.8 \\
\hline N-REM (AB,(-0.5,1),ANES) & 72.8 & $\mathbf{7 9 . 8}$ & 85.9 & 88.0 & 91.2 & 92.7 & 85.1 \\
\hline N-REM (AB,(-3.5,4),ANES) & $\mathbf{7 3 . 9}$ & $\mathbf{7 9 . 8}$ & 85.8 & 87.9 & 91.0 & 92.2 & 85.1 \\
\hline N-REM (AB,SNR-dep.,ANES) & $\mathbf{7 3 . 9}$ & 79.7 & $\mathbf{8 6 . 1}$ & 88.0 & 90.9 & 92.6 & $\mathbf{8 5 . 2}$ \\
\hline \hline GMM-HMM & 49.7 & 57.9 & 67.8 & 73.7 & 80.8 & 82.7 & 68.8 \\
\hline FE-GMM-HMM & 67.2 & 75.9 & 81.1 & 86.4 & 90.7 & 92.0 & 82.2 \\
\hline HMM-FE & 67.0 & 77.0 & 81.8 & 87.0 & 91.2 & 92.4 & 82.7 \\
\hline SC & 76.5 & 81.3 & 88.9 & 90.5 & 92.7 & 93.2 & 87.2 \\
\hline
\end{tabular}

recognition systems such as feature extraction schemes and dictionary sizes are described in (Gemmeke et al., 2013). The FE-GMM-HMM recognizer refers to the baseline NMF system trained on the reverberated data and HMM-regularized FE (HMM-FE) recognizer refers to the proposed system in (Gemmeke et al., 2013). The overcomplete dictionary used by SC, FE-GMM-HMM and HMM-FE recognizers contain 5000 speech and 5000 noise exemplars with exemplar length of 20 frames. The N-REM baseline without ANES uses 400 sniffed noise exemplars only which are extracted from the immediate context of the target utterances (Y1lmaz et al., 2014a).

The keyword recognition accuracies (RA) obtained on the development and test sets of the CHIME-2 data are given in Table 2. The upper panel of each table presents the results provided by the baseline with and without ANES and the proposed recognizers using fixed divergence parameters of $(-0.5,1)$, ($3.5,4)$ and SNR-dependent divergence parameters according to Equation (12) and (13). The best results of the baseline and proposed N-REM systems are given in bold for each SNR level. The lower panels list the results yielded by the comparable recognition systems. The highest performance gains are obtained at the lower SNR levels both for the development and test set. The RAs obtained on the test set using the baseline without ANES are $69.3 \%$ at $-6 \mathrm{~dB}, 76.8 \%$ at $0 \mathrm{~dB}$ and $84.5 \%$ at $3 \mathrm{~dB}$. The second row of the upper panel presents the results provided by the baseline with ANES. The adaptive noise modeling technique improves the noise modeling capabilities providing recognition accuracies of $71.0 \%, 78.9 \%$ and $85.3 \%$ at the same SNR levels. Using the proposed system with SNRdependent divergence parameters, the recognition performance of the proposed setup further increases the recognition performance with RAs of $73.9 \%$ at $-6 \mathrm{~dB}, 79.7 \%$ at $0 \mathrm{~dB}$ and $86.1 \%$ at $3 \mathrm{~dB}$. The total absolute improvements at SNR levels of $-6 \mathrm{~dB}$, $-3 \mathrm{~dB}$ and $0 \mathrm{~dB}$ are $4.6 \%, 2.9 \%$ and $1.6 \%$ respectively. Using SNR-dependent parameters rather than fixed parameters brings only marginal improvements in this scenario.

The RA of the proposed recognizer does not outperform the baseline setup at SNR levels of $6 \mathrm{~dB}$ and $9 \mathrm{~dB}$. The mean RA increases from $84.5 \%$ to $85.8 \%$ on the development set and from $84.1 \%$ to $85.2 \%$ on the test set. The SC recognizer provides comparable performance with N-REM on the development set and slightly better performance on the test set with a RA of $76.5 \%$ at $-6 \mathrm{~dB}, 88.9 \%$ at $0 \mathrm{~dB}$ and 93.2 at $9 \mathrm{~dB}$. The mean RA of the SC recognizer is $86.5 \%$ on the development set and 87.2 on the test set which is the best among all systems. The performance of FE-GMM-HMM and HMM-FE recognizers are similar to each other on both sets for all SNR levels and lower than the SC and N-REM recognizers.

\section{Discussion}

\subsection{Choice of divergence parameters}

The results presented at the lower SNRs of test set A of AURORA-2 and both the test and development set of CHIME2 demonstrate the improved noise robustness of N-REM using the AB divergence. The WER of $14.9 \%$ at the SNR level of 


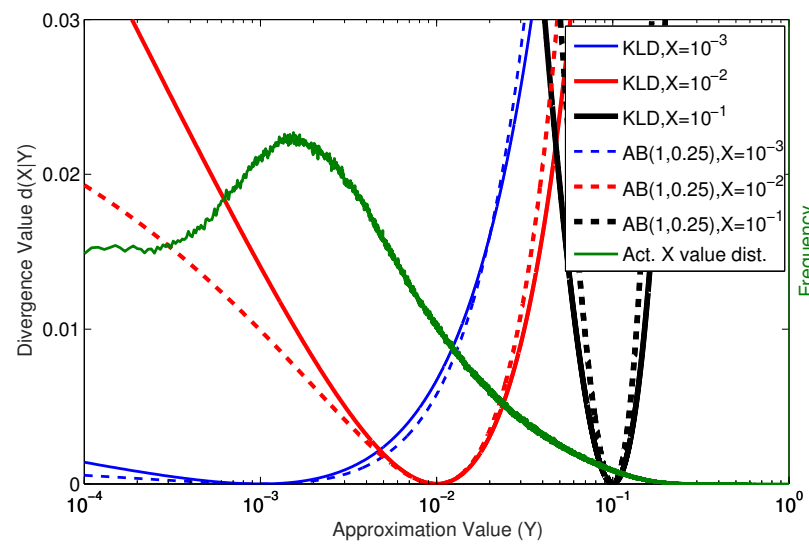

(a) $\mathrm{AB}$ divergence with $(1,0.25)$ (best results for AURORA-2, clean)

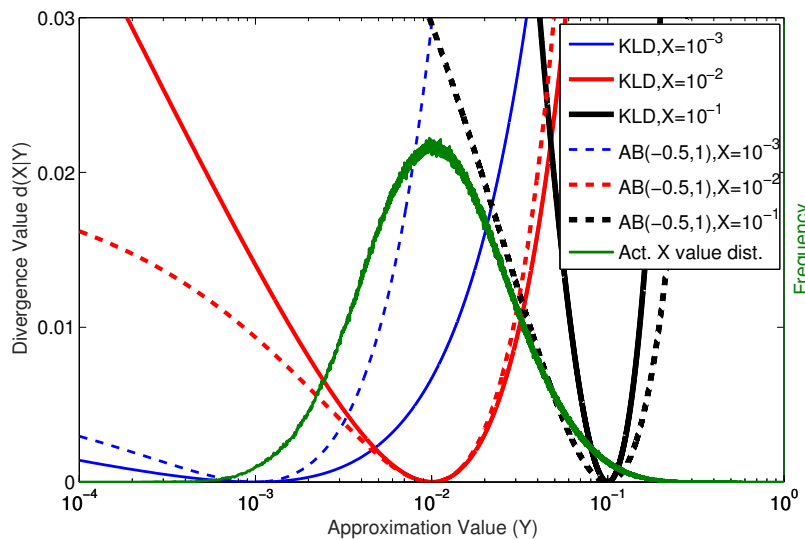

(c) $\mathrm{AB}$ divergence with $(-0.5,1)$ (best results for CHIME-2, $9 \mathrm{~dB}$ )

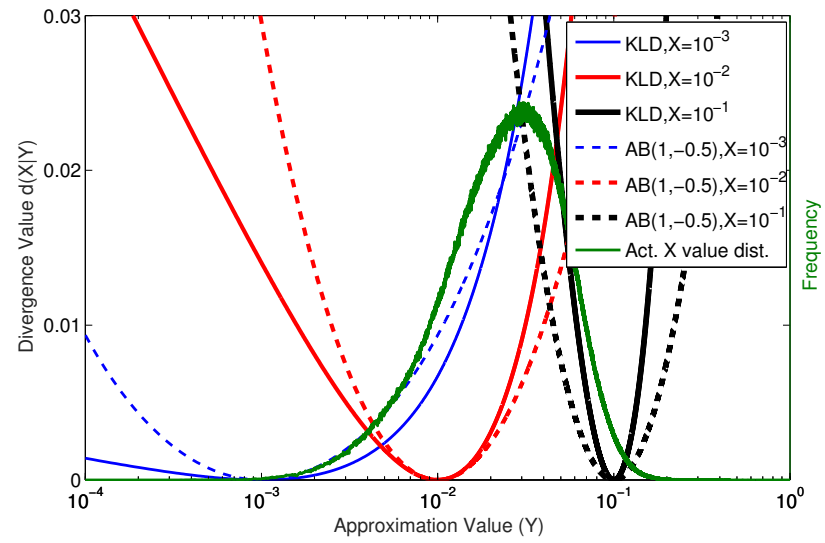

(b) $\mathrm{AB}$ divergence with $(1,-0.5)$ (best results for AURORA-2, $-5 \mathrm{~dB})$

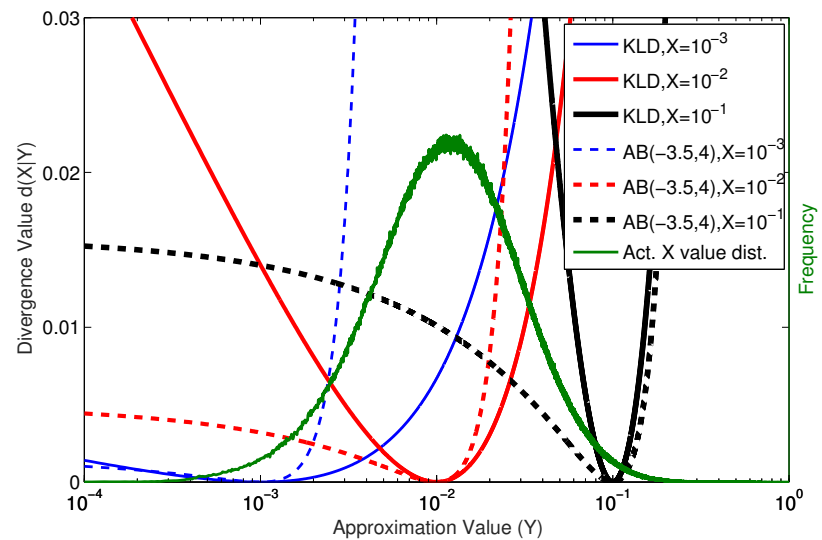

(d) $\mathrm{AB}$ divergence with $(-3.5,4)$ (best results for CHIME-2, $-6 \mathrm{~dB}$ )

Figure 5: Comparison of the divergence value $d(X \mid Y)$ between the AB divergence and generalized KLD for three observation time-frequency cell values $X=$ $[0.001,0.01,0.1]$ and varying approximation values in the range of $0.0001<Y<1$. The green curves show the histogram of occurrence of the actual data values $\mathrm{X}$ on the respective databases.

$-5 \mathrm{~dB}$ of the AURORA-2 data is the best published recognition performance to the best of our knowledge. The proposed recognizer picking an appropriate $(\alpha, \beta)$ value depending on the estimated SNR level performs an accurate speech and noise separation even at the lowest SNR levels. We discuss the reason for the performance gain by visualizing the behavior (regime) of the $\mathrm{AB}$ divergence for several $(\alpha, \beta)$ pairs and comparing them with the ones belonging to the generalized KLD.

Before elaborating on this issue, we revisit some system properties that have been mentioned in the earlier parts of the paper which are relevant to the discussion. Firstly, N-REM uses $l_{2}$-normalized dictionaries where each individual timefrequency cell lies in the range of $[0,1]$. The observation matrices are $l_{2}$-normalized similarly to the dictionaries and all values in the observation matrices are also in the range of $[0,1]$. Secondly, the genuine room noise contaminating the CHIME2 data has different statistical characteristics compared to the noise types in the test set A of AURORA-2. The former noise type has been observed to be less stationary and more spectrotemporally diverse due to the various noise sources in the recording environment such as two adults, two children, TV, kitchen and laundry appliances, foot steps, toys, traffic, birds and so forth (Christensen et al., 2010). We can hence expect that the noise dictionaries for CHIME-2 provide a poorer match to the actual noise spectra compared to the case of AURORA-2. Moreover, the genuine room noise recordings contain reverberation as the recording environments have a $T_{60}=0.3$ seconds.
Taking this information into account, we discuss the reasons of the performance gain by comparing the weighting and scaling behaviors of the generalized KLD and AB divergence with divergence parameters that provided the best results at the lowest and highest SNR level of both databases in Figure 5. The upper figures show the behavior of the $A B$ divergence with $(1,0.25)$ and $(1,-0.5)$ providing lower WERs on the clean test set and the test set at SNR level of $-5 \mathrm{~dB}$ of AURORA-2 respectively. We plot the divergence value for three different values of a time-frequency cell from an observation vector (denoted by $\mathrm{X}$ in the figure) with varying approximation values (denoted by $\mathrm{Y}$ in the figure). However, the recognition framework is invariant to scaling of the divergence, since scaling will not change the ranking of the recognition hypotheses. To avoid false interpretation of the divergence plots, we can therefore scale each $A B$ divergence plot. We choose the scaling factor such that the local behavior of the AB divergence is the same as the local behavior of the KLD at the medium reference data value $(X)$ of 0.01 , i.e. KLD and $\mathrm{AB}$ divergence have the same curvature at the reference point. We choose a reference point of 0.01 because the probability density is high for both databases. This can be verified from the distribution of real time-frequency cell values after the $l_{2}$-normalization as depicted with the green curve in Figure 5. It is worth pointing out that the density plot belonging to the clean speech of AURORA-2 is obtained only from the segments that contain speech, i.e. silence frames are discarded. 


\subsubsection{AURORA-2}

Firstly, we observe that the AB divergence with $(1,0.25)$ provides good accuracy for the clean test set of AURORA-2. From Figure 5a, it is clearly seen that the $\mathrm{AB}$ divergence downweights the smaller cells which contain little or no energy and puts more emphasis on the large cells, i.e. the spectral peaks. The very high SNR observed in the clean AURORA-2 data is reflected in the green density plot, where we observe a substantial fraction of the data with less energy than $40 \mathrm{~dB}$ below the spectral peaks. To approximate such small spectral values accurately with a linear combination of atoms should not matter. Hence, there is no harm to reduce the penalty of underestimations of small values (red and blue curves below $\mathrm{Y}=0.01$ in Figure 5a).

The noise robustness of N-REM depends on how well the noise exemplars model the actual noise conditions and how accurate the divergence weights the approximation error of time-frequency cells that define the characteristics of the noise source. This is vital for accurate separation of speech and noise. The best performance at SNR level of $-5 \mathrm{~dB}$ of AURORA-2 is obtained using the divergence parameters $(1,-0.5)$. The divergence with $(1,-0.5)$ is equally far from the generalized KLD (AB divergence with parameters $(1,0)$ ) and the Itakura-Saito distance (AB divergence with parameters $(1,-1)$ ) on the $\alpha=1$ line which is equal to the Beta divergence as a special case of the $\mathrm{AB}$ divergence. In this regard, it is a compromise between the generalized KLD and the Itakura-Saito distance which has been shown to be effective on source separation tasks using audio power spectrograms (Févotte et al., 2009). The value of the divergence with $(1,-0.5)$ is logarithmically proportional to the scale of the input, i.e. $d_{A B}^{(1,-0.5)}(k \cdot \mathbf{y}, k \cdot \hat{\mathbf{y}})=\sqrt{k} \cdot d_{A B}^{(1,-0.5)}(\mathbf{y}, \hat{\mathbf{y}})$, while the generalized KLD cost is linearly proportional and the Itakura-Saito distance is scale-invariant (Virtanen, 2007; Févotte et al., 2009). As a result, the smaller observations contribute more to the total cost using this divergence compared to the generalized KLD, while the larger observations contribute still more than the smaller ones unlike the Itakura-Saito distance.

The behavior of the $\mathrm{AB}$ divergence with $(1,-0.5)$ is shown in Figure 5b. From this figure, it can be seen that the divergence upweights the approximation errors of small time-frequency cells (e.g. $\mathrm{X}=0.001$ in the figure), compared to the generalized KLD. Looking at the data distribution (green), we see that this has no relevance, as actual data points in this order of magnitude are hardly observed. Medium $(X=0.01)$ to large $(X=0.1)$ data values should now be realized exactly during the approximation as both over and underestimation are penalized. Since the noise dictionaries are highly accurate for this database, this is indeed a very good strategy to obtain an accurate signal decomposition in terms of speech and noise. Eventually, a few noise exemplars that resemble the actual noise component are selected from the noise dictionary providing an accurate separation and thus a high recognition performance. This result matches up with the recognition results presented in (Y1lmaz et al., 2014b) where a grid search on the AB plane has been performed to find the most appropriate divergence parameters at the lower SNRs of CHIME-2. The best recognition results are obtained on the $\alpha+\beta=0.5$ line which passes through the $(1,-0.5)$ point.

After the discussion on the recognition performance in the matched noise scenario, we discuss the results obtained on the test set B of AURORA-2 which contains mismatched noise types, i.e. noise types that are not available in the training data. Despite exemplar-based modeling being quite effective in the case of matched noise, there is a performance gap between matched and mismatched noise scenarios for all exemplarbased techniques. Using the $\mathrm{AB}$ divergence with $(1,-0.5)$ does not help much at lower SNR levels as there is a more fundamental problem, i.e. mismatched noise exemplars do not provide an accurate model in the high feature dimensional space. As a result, the recognizer has the tendency to explain the noise component using speech exemplars and noisy mixtures often cannot be separated into its components resulting in an inferior recognition performance. This gap is larger for N-REM than for SC and FE-GMM-HMM since N-REM dictionaries contain fewer exemplars. This results in a lower probability of having a suitable noise exemplar in the combined dictionaries with a similar spectral content with the unseen noise types. The use of exemplars is most applicable in scenarios where the expected noise types can be predicted or when some noise exemplars can be readily obtained from the environment as in the CHIME-2 data.

\subsubsection{CHIME-2}

In case of CHIME-2, the recognition performance also benefits from using smaller $\alpha$ values on the $\alpha+\beta=0.5$ line. The lower figures in Figure 5 show the behavior of the AB divergence with $(-0.5,1)$ and $(-3.5,4)$ providing best results on the test set at SNR level of $9 \mathrm{~dB}$ and $-6 \mathrm{~dB}$ respectively. On CHIME-2, we observe that the optimal divergence choice downweights underestimations for small data values (dashed blue and red curves in the lower panel of Figure 5) compared to the $\mathrm{AB}$ divergence with $(1,-0.5)$ (dashed blue and red curves in Figure 5b). At the lower SNR (Figure 5d), this is even the case for the largest of spectral values (black curves). Since the noise dictionaries are not expected to be very accurate for CHIME-2, while speech dictionaries are, the noisy data is best explained based on the time-frequency cell that are dominated by speech, and a divergence metric putting more emphasis on these speech-dominated cells is a sensible choice. This is in line with missing data techniques for speech recognition (Cooke et al., 2001), which constrain the clean speech model to be less than the observed noisy speech for time-frequency cells dominated by noise, while the clean speech model should be equal to the noisy observations for time-frequency cells dominated by speech.

At higher SNR (Figure 5c), large spectral values mostly contain speech and the approximation should match the noisy observation at these reliable time-frequency cells, which is indeed expressed by the black curve in Figure 5c. The small timefrequency cells are most likely dominated by noise and reverberation, so underestimation of the smaller cells to match the reliable spectral peaks should be less penalized compared to the $\mathrm{AB}$ divergence with $(1,-0.5)$. At low SNRs, large spectral 
values are also often dominated by noise and it is hence helpful to allow not to penalize the underestimation of large spectral values as well which is expressed by the black dashed curve in Figure 5d. In this scenario, there is no clear evidence for reliable time-frequency cells that can be approximated accurately by the speech dictionaries. Thus, the most accurate approximation approach in the absence of accurate noise dictionaries is to limit the overestimations for all observed data values. In practice, the approximations provided by the $\mathrm{AB}$ divergence with $(-3.5,4)$ contain less overestimations due to the high penalty which avoids severe overestimations of smaller time-frequency cells to model the spectral peaks using inaccurate noise exemplars. Moreover, the approximation error due to the underestimations has an upper bound for each real data value $(X=0.001$, $\mathrm{X}=0.01, \mathrm{X}=0.1$ ) which is visible from the dashed curves in Figure $5 \mathrm{~d}$ at the approximation values that are smaller than the real data values. This results in a more balanced contribution of approximation errors to the total RE from each time-frequency cell.

\subsubsection{Summary}

Based on these results, it can be summarized that the using the $\mathrm{AB}$ divergence with parameters on the $\alpha+\beta=0.5$ line is found to be effective for separation and recognition of noisy speech signals represented in mel-scaled magnitude spectral domain. Depending on the quality of the noise model, different values of $\alpha$ and $\beta$ can chosen to further improve the recognition accuracy. For smaller $\alpha$ values, the proposed technique behaves in line with the missing data techniques by limiting the overestimations of the noisy spectra which provides better recognition performance in case of poor noise modeling, e.g. due to severe non-stationarity. Larger $\alpha$ values enforce more precise approximation of both small and large observations yielding improved recognition accuracy in the case of accurate speech and noise models.

\subsection{ANES with genuine room noise}

The performance of the adaptive noise modeling technique ANES has been investigated in a more realistic scenario in which noisy utterances are contaminated with genuine room noise. The results presented in Table 2 show that ANES combined with noise sniffing can model the genuine room noise more accurately compared to noise sniffing only. These results demonstrate the effective noise modeling of ANES achieved by picking a small number of noise exemplars which have similar characteristics to the actual noise contaminating the target utterance.

\subsection{Computational Effort}

The computational bottleneck of the proposed framework is the multiplicative update rule given in Equation (3). The evaluation of (3) for all combined dictionaries is performed in parallel for each length and is accelerated by using a GPU. For all experiments, we have used a GeForce GTX 980 GPU on a cluster computer with 16 Intel Xeon processor E5620 (12M Cache, $2.40 \mathrm{GHz}$ ) and $99 \mathrm{~GB}$ memory. To quantify the simulation times for each task, we have timed the recognition processes in MATLAB for each utterance and averaged the simulation time per utterance over each test set.

The SNR-dependent noise dictionaries yield different simulation times at each SNR level in AURORA-2 database. At -5 $\mathrm{dB}$, the recognizer uses the highest number of noise exemplars, hence, the longest simulation times are expected at this SNR level. After averaging over a set containing 250 utterances with a mean duration of 1.7 seconds, the average recognition time is found to be 16.8 seconds with a standard deviation of $4.7 \mathrm{sec}-$ onds using the generalized KLD with induced sparsity and 26.6 seconds with a standard deviation of 7.6 seconds using the $\mathrm{AB}$ divergence. For higher SNRs, the SNR-dependent noise modeling has reduced the simulation times as the combined dictionaries contain less noise exemplars. On clean speech, the average recognition time reduces to 9.2 seconds with a standard deviation of 2.4 seconds using the generalized KLD with induced sparsity and 16.3 seconds with a standard deviation of $3.7 \mathrm{sec}-$ onds using the $\mathrm{AB}$ divergence. These average recognition times include the time required for the preprocessing, which takes 1.5 seconds in average with a standard deviation of 0.3 seconds.

On the CHIME-2 data, the average recognition time is obtained by averaging the recognition time over 600 test utterances. The mean duration of these utterances is 1.8 seconds and the average recognition time is 10.4 seconds with a standard deviation of 1.8 seconds using the generalized KLD with induced sparsity and 12.9 seconds with a standard deviation of $2.1 \mathrm{sec}-$ onds using the $\mathrm{AB}$ divergence. These average recognition times include the time required for the preprocessing, which takes 1.6 seconds in average with a standard deviation of 0.2 seconds.

\section{General Discussion and Concluding Remarks}

In this paper, we have aimed to improve the noise robustness of our noise robust exemplar matching approach by adopting the $\mathrm{AB}$ divergence which has a higher degree of freedom with two parameters compared to the generalized KLD. Various well-known distance/divergence measures such as the squared Euclidean distance, generalized Kullback-Leibler divergence, Itakura-Saito divergence and Hellinger distance are special cases of the $\mathrm{AB}$ divergence for different $(\alpha, \beta)$ values. By adjusting these parameters on the development data, the divergence is tailored for the representation of speech and noise for the best separation of the noisy mixtures. Applying the multiplicative update rules proposed for this new divergence in $(\mathrm{Ci}-$ chocki et al., 2011), the noisy utterances are modeled as a linear combination of exemplars that are organized in multiple dictionaries based on their duration and class. The presented recognition results have confirmed the improved noise robustness of the $\mathrm{AB}$ divergence compared to the conventional generalized KLD. After presenting the results, we have provided insight into the choice for the $(\alpha, \beta)$ pairs along the $\alpha+\beta=0.5$ line depending on the parameters such as SNR and quality of the noise dictionaries.

Focused on investigating the noise robustness of a pure exemplar matching-based recognizer using this flexible divergence measure, we prefer not adopting a hybrid approach (cf. 
(De Wachter et al., 2007)) in the scope of this paper, which would immediately enhance the recognition performance at higher SNR levels. In such a setting, the acoustic scores obtained from both streams can be combined to benefit from the noise robustness of exemplar-based acoustic modeling and better discrimination of the statistical models such as complex GMM distributions in conjunction with MFCC features or DNNs. One possible future direction is to develop a robust way of combining the acoustic scores that are obtained using different models, such as the reconstruction errors of exemplar matching and the likelihoods of the GMMs or "pseudolikelihoods" of the DNNs.

Another way to improve the performance at higher SNR levels is to employ a similar strategy to the FE-GMM-HMM recognizer, i.e. using the proposed technique for speech enhancement in the front end and perform recognition on the enhanced speech features using the statistical models (cf. (Gemmeke et al., 2011a)). Recently, an alternative FE-GMM-HMM approach using coupled dictionaries has been shown to improve the recognition performance (Baby et al., 2014). Another future work includes exploring the speech enhancement performance of N-REM to see if more accurate recognition can be achieved on the enhanced features using powerful statistical approaches such as GMM/HMM or DNNs.

The proposed recognizer will also benefit from estimating the divergence parameters without any prior investigation, as the divergence parameters providing the best performance depends highly on the characteristics of noisy mixtures (Cichocki et al., 2011). An asymmetric clustering algorithm using the AB divergence has been recently proposed which estimates the divergence parameters based on the within-cluster variances (Olszewski and Šter, 2014). A systematic and computationally efficient way of estimating the divergence parameters from the training data would reduce the computational burden due to the initial search for suitable values.

Finally, the noise robust exemplar matching framework applied on medium to large vocabulary speech is yet another crucial step to take in the future. Its main advantage is that the exemplars model speech units, which should scale better than the long, fixed-length exemplars employed in the previous SR approaches. In this work, the speech exemplars have been chosen to represent half-words. Considering the dimensionality and computational restrictions, the same framework using exemplars associated with more general subword units such as phones or syllables could be applied to a medium or large vocabulary task. Only the current decoding scheme would need to be redesigned in a way that it will incorporate a language model combined with the acoustic costs, but for this it could largely rely on existing exemplar matching frameworks (De Wachter et al., 2007).

\section{Acknowledgements}

This work has been supported by the KU Leuven research grant OT/09/028 (VASI) and IWT-SBO Project 100049 (ALADIN).

\section{References}

Aradilla, G., Vepa, J., Bourlard, H., 2005. Improving speech recognition using a data-driven approach. In: Proc. INTERSPEECH. Lisbon, Portugal, pp. 3333-3336.

Baby, D., Virtanen, T., Gemmeke, J. F., Barker, T., Van hamme, H., Dec. 2014. Exemplar-based noise robust automatic speech recognition using modulation spectrogram features. In: IEEE SLT Workshop. South Lake Tahoe, USA, pp. 519-524.

Benzeghiba, M., De Mori, R., Deroo, O., Dupont, S., Erbes, T., Jouvet, D., Fissore, L., Laface, P., Mertins, A., Ris, C., Rose, R., Tyagi, V., Wellekens, C., 2007. Automatic speech recognition and speech variability: A review. Speech Communication 49 (1011), 763-786.

Bourlard, H., Hermansky, H., Morgan, N., 1996. Towards increasing speech recognition error rates. Speech Communication 18 (3), 205 - 231.

Christensen, H., Barker, J., Ma, N., Green, P., Sept. 2010. The CHiME corpus: a resource and a challenge for computational hearing in multisource environments. In: Proc. INTERSPEECH. Makuhari, Japan.

Cichocki, A., Cruces, S., Amari, S.-I., 2011. Generalized alpha-beta divergences and their application to robust nonnegative matrix factorization. Entropy $13(1), 134-170$.

Cichocki, A., Zdunek, R., Amari, S., 2006. Csiszár's divergences for nonnegative matrix factorization: family of new algorithms. In: Proc. of the 6th International Conference on Independent Component Analysis and Blind Signal Separation. pp. 32-39.

Cooke, M., Barker, J., Cunningham, S., Shao, X., 2006. An audio-visual corpus for speech perception and automatic speech recognition. Journal of the Acoustical Society of America 120 (5), 2421-2424.

Cooke, M., Green, P., Josifovski, L., Vizinho, A., 2001. Robust automatic speech recognition with missing and unreliable acoustic data. Speech Communication 34 (3), 267-285.

De Wachter, M., Demuynck, K., Van Compernolle, D., Wambacq, P., Sep. 2003. Data-driven example based continuous speech recognition. In: Proc. EUROSPEECH. pp. 1133-1136.

De Wachter, M., Matton, M., Demuynck, K., Wambacq, P., Cools, R., Van Compernolle, D., May 2007. Template-based continuous speech recognition. IEEE Transactions on Audio, Speech, and Language Processing 15 (4), 1377-1390

Deselaers, T., Heigold, G., Ney, H., 2007. Speech recognition with state-based nearest neighbour classifiers. In: Proc. INTERSPEECH. Antwerp, Belgium, pp. 2093-2096.

Donoho, D., Stodden, V., 2004. When does non-negative matrix factorization give a correct decomposition into parts? In: Advances in Neural Information Processing Systems 16. MIT Press, Cambridge, MA.

Févotte, C., Bertin, N., Durrieu, J.-L., Mar. 2009. Nonnegative matrix factorization with the Itakura-Saito divergence: With application to music analysis. Neural Comput. 21 (3), 793-830.

Gemmeke, J., Virtanen, T., Hurmalainen, A., Sept. 2011a. Exemplar-based sparse representations for noise robust automatic speech recognition. IEEE Transactions on Audio, Speech, and Language Processing 19 (7), 20672080 .

Gemmeke, J. F., Hurmalainen, A., Virtanen, T., 2013. HMM-regularization for NMF-based noise robust ASR. In: 2nd International Workshop on Machine Listening in Multisource Environments. pp. 47-52.

Gemmeke, J. F., Van hamme, H., Sept. 2012. Advances in noise robust digit recognition using hybrid exemplar-based techniques. In: Proc. INTERSPEECH. Portland, USA, pp. 1-4.

Gemmeke, J. F., Virtanen, T., 2010. Artificial and online acquired noise dictionaries for noise robust ASR. In: Proc. INTERSPEECH. pp. 2082-2085.

Gemmeke, J. F., Virtanen, T., Hurmaleinen, A., Sept. 2011b. Exemplar-based speech enhancement and its application to noise-robust automatic speech recognition. In: International Workshop on Machine Listening in Multisource Environments. pp. 53-75.

Golipour, L., O'Shaughnessy, D., Apr. 2009. Context-independent phoneme recognition using a k-nearest neighbour classification approach. In: Proc. ICASSP. pp. 1341-1344.

Heigold, G., Nguyen, P., Weintraub, M., Vanhoucke, V., 2012. Investigations on exemplar-based features for speech recognition towards thousands of hours of unsupervised, noisy data. In: Proc. ICASSP. Kyoto, Japan, pp. 44374440.

Hirsch, H. G., Pearce, D., Sept. 2000. The Aurora experimental framework for the performance evaluation of speech recognition systems under noisy 
conditions. In: Proc. ISCA Tutorial and Research Workshop ASR2000. pp. 181-188.

Hirsch, H. G., Pearce, D., Sept. 2006. Applying the Advanced ETSI frontend to the Aurora-2 task. Tech. rep., version 1.1.

Hoyer, P., 2002. Non-negative sparse coding. In: IEEE Workshop on Neural Networks for Signal Processing. pp. 557-565.

Hoyer, P. O., Dec. 2004. Non-negative matrix factorization with sparseness constraints. J. Mach. Learn. Res. 5, 1457-1469.

Hurmalainen, A., Gemmeke, J., Virtanen, T., May 2011. Non-negative matrix deconvolution in noise robust speech recognition. In: Proc. ICASSP. pp. 4588-4591.

Kanevsky, D., Sainath, T., Ramabhadran, B., Nahamoo, D., 2010. An analysis of sparseness and regularization in exemplar-based methods for speech classification. In: Proc. INTERSPEECH. Makuhari, Chiba, Japan, pp. 2842 2845.

King, B., Fevotte, C., Smaragdis, P., 2012. Optimal cost function and magnitude power for NMF-based speech separation and music interpolation. In: IEEE International Workshop on Machine Learning for Signal Processing (MLSP). pp. 1-6.

Lee, D. D., Seung, H. S., 2000. Algorithms for non-negative matrix factorization. In: Advances in Neural Information Processing Systems 13. MIT Press, pp. 556-562.

Ney, H., Apr 1984. The use of a one-stage dynamic programming algorithm for connected word recognition. IEEE Transactions on Acoustics, Speech and Signal Processing 32 (2), 263-271.

Ney, H., Ortmanns, S., Sep 1999. Dynamic programming search for continuous speech recognition. IEEE Signal Processing Magazine 16 (5), 64-83.

Olszewski, D., Šter, B., 2014. Asymmetric clustering using the alpha-beta divergence. Pattern Recognition 47 (5), 2031-2041.

Povey, D., Zhang, X., Khudanpur, S., 2015. Parallel training of dnns with natural gradient and parameter averaging. In: ICLR Workshop. URL http: //arxiv .org/abs/1410.7455

Raj, B., Virtanen, T., Chaudhuri, S., Singh, R., 2010. Non-negative matrix factorization based compensation of music for automatic speech recognition. In: Proc. INTERSPEECH. Makuhari, Chiba, Japan, pp. 717-720.

Sainath, T. N., Ramabhadran, B., Nahamoo, D., Kanevsky, D., Sethy, A., Sept. 2010. Sparse representations features for speech recognition. In: Proc. INTERSPEECH. pp. 2254-2257.

Sainath, T. N., Ramabhadran, B., Nahamoo, D., Kanevsky, D., Van Compernolle, D., Demuynck, K., Gemmeke, J. F., Bellegarda, J. R., Sundaram, S., Nov. 2012. Exemplar-based processing for speech recognition: An overview. IEEE Signal Processing Magazine 29 (6), 98-113.

Sakoe, H., Chiba, S., 1971. A dynamic programming approach to continuous speech recognition. In: Proceedings of the Seventh International Congress on Acoustics. Vol. 3. Budapest, Hungary, pp. 65-69.

Seppi, D., Van Compernolle, D., Sept. 2010. Data pruning for template-based automatic speech recognition. In: Proc. INTERSPEECH. Makuhari, Chiba, Japan, pp. 985-988

Smaragdis, P., 2007. Convolutive speech bases and their application to supervised speech separation. IEEE Transactions on Audio, Speech, and Language Processing 15 (1), 1-12.

Smaragdis, P., Brown, J. C., 2003. Non-negative matrix factorization for polyphonic music transcription. In: IEEE Workshop on Applications of Signal Processing to Audio and Acoustics. pp. 177-180.

Sun, X., Zhao, Y., Aug. 2011. New methods for template selection and compression in continuous speech recognition. In: Proc. INTERSPEECH. Florence, Italy, pp. 985-988.

Sun, Y., Gemmeke, J. F., Cranen, B., ten Bosch, L., Boves, L., 2014. Fusion of parametric and non-parametric approaches to noise-robust ASR. Speech Communication $56(0), 49-62$.

Sundaram, S., Bellegarda, J. R., 2012. Latent perceptual mapping with datadriven variable-length acoustic units for template-based speech recognition. In: Proc. ICASSP. pp. 4125-4128.

Tan, Q. F., Narayanan, S. S., May 2012. Novel variations of group sparse regularization techniques with applications to noise robust automatic speech recognition. IEEE Transactions on Audio, Speech, and Language Processing 20 (4), 1337-1346.

Vincent, E., Barker, J., Watanabe, S., Le Roux, J., Nesta, F., Matassoni, M., May 2013. The second 'CHiME' speech separation and recognition challenge: Datasets, tasks and baselines. In: Proc. ICASSP. Vancouver, Canada, pp. $126-130$.
Virtanen, T., March 2007. Monaural sound source separation by nonnegative matrix factorization with temporal continuity and sparseness criteria. IEEE Transactions on Audio, Speech, and Language Processing 15 (3), 10661074.

Yılmaz, E., Gemmeke, J. F., Van hamme, H., Sept. 2013a. Exemplar selection techniques for sparse representations of speech using multiple dictionaries. In: Proc. EUSIPCO. Marrakesh, Morocco, pp. 1-5.

Yılmaz, E., Gemmeke, J. F., Van hamme, H., June 2013b. Noise-robust automatic speech recognition with exemplar-based sparse representations using multiple length adaptive dictionaries. In: 2nd International Workshop on Machine Learning in Multisource Environments (CHIME). Vancouver, Canada, pp. 39-43.

Yılmaz, E., Gemmeke, J. F., Van hamme, H., Aug. 2014a. Noise robust exemplar matching using sparse representations of speech. IEEE/ACM Transactions on Audio, Speech, and Language Processing 22(8), 1306-1319.

Yılmaz, E., Gemmeke, J. F., Van hamme, H., May 2014b. Noise-robust speech recognition with exemplar-based sparse representations using alpha-beta divergence. In: Proc. ICASSP. Florence, Italy, pp. 5539-5543.

Zhang, X., Trmal, J., Povey, D., Khudanpur, S., May 2014. Improving deep neural network acoustic models using generalized maxout networks. In: Proc. ICASSP. pp. 215-219. 